\title{
Originalarbeit
}

Obere Extremität 2020 $15: 103-110$ https://doi.org/10.1007/s11678-020-00574-0 Received: 3 February 2020

Accepted: 16 April 2020

Published online: 14 May 2020

(c) The Author(s) 2020

Alexander Ellwein ${ }^{1,2} \cdot$ Martin Jaeger $^{3} \cdot$ Christine Voigt $^{1} \cdot$ Jan Christoph Katthagen ${ }^{4} \cdot$ Christian Schoepp ${ }^{5} \cdot$ Karl-Heinz Frosch $^{6} \cdot$ Helmut Lill $^{1} \cdot$ Gunnar Jensen ${ }^{1}$

'Department for Orthopaedic Surgery and Traumatology, DIAKOVERE Friederikenstift, Hannover, Germany ${ }^{2}$ Department for Orthopaedic Surgery, Medical School Hannover, DIAKOVERE Annastift, Hannover, Germany

${ }^{3}$ Department of Orthopaedic and Trauma Surgery, University Medical Center Freiburg, Freiburg, Germany ${ }^{4}$ Department of Trauma, Hand and Reconstructive Surgery, University Hospital Münster, Münster, Germany

${ }^{5}$ Departement of Orthopedic and Trauma Surgery, Berufsgenossenschaftliche Unfallklinik Duisburg, Duisburg, Germany

${ }^{6}$ Department of Trauma, Hand and Reconstructive Surgery, University Medical Center HamburgEppendorf, Hamburg, Germany

\section{Arthroscopically assisted stabilization versus hook plate fixation for chronic acromioclavicular joint separation}

\section{Short-term follow-up of a prospective multicenter study}

which may result in satisfactory outcomes [5-11]. In chronic cases, the torn ligaments lose their healing potential over time, due to degenerative changes. Therefore, the ligaments must be biologically augmented by a tendon graft. An interval of more than 21 days after trauma is commonly defined as the border between acute and chronic injuries [12]. Still, among shoulder surgeons, this time interval remains controversial.

Surgical treatment of chronic AC joint instability can be performed using either open techniques, such as with a hook plate, or with arthroscopically assisted techniques [10, 12-14]. Due to the potential detection of concomitant intraarticular injuries and the advantages of a minimally invasive approach, the number of arthroscopically assisted techniques is increasing [15].

Nonetheless, data on clinical results and recommended techniques for treating chronic AC joint instability are limited. The aim of this study was to evalu- ate clinical and radiological results after treatment for chronic AC joint instability. We hypothesized that (1) operative treatment would significantly improve patients' outcome in short-term followup (2) without a significant difference between the two groups.

\section{Materials and methods}

\section{Patient selection}

The Trauma Committee of the Society for Arthroscopy and Joint Surgery (Gesellschaft für Arthroskopie und Gelenkchirurgie, AGA) initiated this prospec tive multicenter study. Five level I trauma centers participated and included patients. All patients with chronic highgrade AC joint instability after failed operative or non-operative treatment were included in the study and prospectively monitored. High-grade AC joint instability was defined as Rockwood type III-V. A minimum interval of ing response of the ruptured ligaments, 

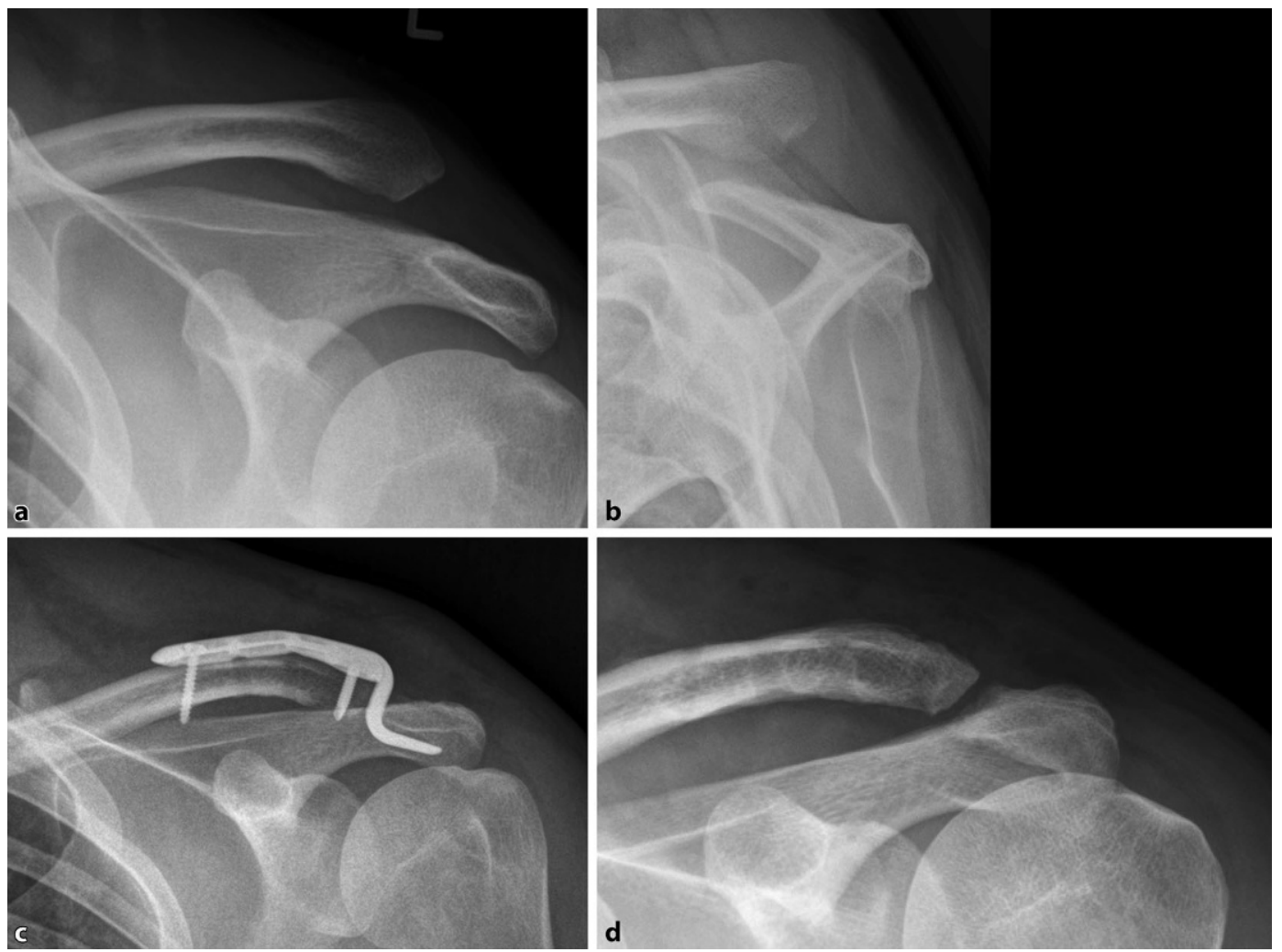

Fig. $1<$ a Rockwood type $\mathrm{V}$ lesion in the anteroposterior view with b horizontal instability in the Alexander view. c Hook plate stabilization with additional tendon graft augmentation. $\mathbf{d}$ Result at final follow-up

3 months between trauma and index surgery was required to define a chronic situation. All patients had to be between 18 and 65 years of age. Patients with pre-existing pathologies beside AC joint injury and related surgery which limited shoulder function, strength, or movement were excluded.

\section{Surgical technique}

To restore stability, patients were treated either openly, using hook plate fixation (- Fig. 1), or by an arthroscopically assisted technique using a flip-button device (• Fig. 2). An additional autologous gracilis tendon graft for coracoclavicular (CC) and AC ligament reconstruction was included in each technique. The arthroscopically assisted technique was performed according to the technique published by Scheibel et al. [16]. The CC flip-button device (TightRope, Arthrex, Naples, FL, USA) was inserted through a 4-mm drill hole. Two clavicular 4-mm drill holes positioned at the former CC ligaments and one through the coracoid were drilled for augmentation of the CC ligaments. The graft was pulled through and fixed with two tenodesis screws (each $4 \times 10 \mathrm{~mm}$ ) at the clavicle. The end of the graft was used for AC augmentation. An acromial 4-mm drill hole was created, ascending from lateral and pointing to the proximal acromial edge of the AC joint. The graft was pulled through the acromion, shuttled back subcutaneously, and sutured to the remaining end of the graft. In the hook plate group, the graft was also pulled through two 4-mm drill holes in the clavicle, but just wrapped around the coracoid without an additional drill hole. The ends of the graft were sutured above the clavicle. The remaining ends of the graft were then sutured to the acromion in terms of an AC augmentation.

\section{Rehabilitation protocol}

During the first 6 weeks after hook plate implantation, motion was limited to $90^{\circ}$ flexion and abduction. From the seventh week on, a free range of motion was allowed. Implant removal was performed 3 months after surgery. In the arthroscopic group, shoulders were protected in a sling for 6 weeks. During the first
3 weeks, patients were allowed only passive motion up to a flexion and abduction of $45^{\circ}$. From the fourth week, patients performed active/assistive motions up to a flexion and abduction of $45^{\circ}$, and up to $60^{\circ}$ in weeks 5 and 6 . From the seventh week on, free motion was allowed. After 12 weeks, muscle strengthening exercises were initiated in both groups.

\section{Radiographic outcome measures}

Preoperatively, all patients were graduated according to the Rockwood classification using bilateral anteroposterior stress views of the AC joint. According to Rockwood, an increase in CC distance of 25 to $100 \%$ is defined as a Rockwood type III injury, an increase of more than $100 \%$ as type V $[17,18]$. Bilateral Alexander views and an axillary view of the affected shoulder were used for detecting static or dynamic horizontal instability [19]. A difference of more than one clavicle shaft was defined as horizontally instable. Further radiographs were performed postoperatively and at a follow-up. The changes in CC distance were compared preoperatively, postoper- 
Obere Extremität 2020 · 15:103-110 https://doi.org/10.1007/s11678-020-00574-0

(c) The Author(s) 2020

A. Ellwein · M. Jaeger · C. Voigt · J. C. Katthagen · C. Schoepp · K.-H. Frosch · H. Lill · G. Jensen

\section{Arthroscopically assisted stabilization versus hook plate fixation for chronic acromioclavicular joint separation. Short-term follow-up of a prospective multicenter study}

\section{Abstract}

Background. Chronic acromioclavicular (AC) joint instability may result after failed surgical or non-operative treatment of acute AC joint dislocation. Data on clinical outcomes of chronic AC joint instability are limited; thus, no gold standard surgical technique exists. Objective. This study aimed to evaluate clinical and radiological results after arthroscopically assisted or hook plate stabilization of chronic type III-V AC joint instability. We hypothesized that (1) operative treatment would improve outcomes in short-term follow-up (2) without a difference between the groups.

Materials and methods. Five level I trauma centers prospectively included patients in this non-randomized multicenter study. Patients had undergone reconstruction of the coracoclavicular (CC) and AC ligaments with an anatomic hamstring autograft. Graft healing was supported by arthroscopically assisted AC joint stabilization using a flip button or by a hook plate. Inclusion criteria were chronic high-grade (Rockwood III-V) AC joint instability and failed surgical or non-operative treatment after a trial of conservative management measures $\geq 12$ weeks. Functional outcome measures (Subjective Shoulder Value, Constant Score, Taft Score, Acromioclavicular Joint Instability Score) were recorded preoperatively and at follow-up. Radiographs were performed preand postoperatively, and at follow-up. Results. A total of 43 patients were included (mean age $44 \pm 12$ years, range $18-64 ; 17$, $40 \%$, Rockwood III and 26, 60\%, Rockwood V; $32,74 \%$, arthroscopic and $11,26 \%$, hook plate; 35 available for follow-up; mean followup $12 \pm 3$ months, range $6-20$ ). All clinical outcome measures improved significantly $(p<0.001)$, with no differences between the groups. CC distance improved significantly from pre- to postoperative $(19 \pm 5 \mathrm{~mm}$ to $8 \pm 2 \mathrm{~mm} ; p<0.001)$ but was lost at followup $(13 \pm 4 \mathrm{~mm} ; p<0.001)$. Two patients were revised after arthroscopic stabilization. All patients with hook plate stabilization required additional surgery for implant removal. Conclusion. Surgical treatment for symptomatic chronic AC joint instability improves clinical outcomes in short-term follow-up. Functional results of arthroscopically assisted and hook plate stabilization are comparable. Nonetheless, some loss of reduction can be observed. Level of evidence: IV.

Keywords Joint dislocation · Trauma · Grafts · Shoulder . Ligaments

\section{Therapie chronischer Akromioklavikulargelenkinstabilitäten mittels arthroskopisch assistierter Stabilisierung oder Hakenplatte. Kurzzeit-Follow-up einer prospektiven Multizenterstudie}

\section{Zusammenfassung}

Hintergrund. Schlägt die operative oder konservative Therapie in der Behandlung akuter akromioklavikulärer (AC-)Luxationen fehl, können daraus chronische Instabilitäten resultieren. Bisher ist die Datenlage zu den klinischen Ergebnissen bei chronischen AC-Gelenkinstabilitäten limitiert. Ein Goldstandard für die optimale Versorgung fehlt bisher.

Ziel. Ziel dieser Studie war es, die klinischen und radiologischen Ergebnisse nach Stabilisierung chronischer AC-Gelenkinstabilitäten vom Typ III-V mittels arthroskopisch gestützter Technik oder Hakenplatte zu evaluieren. Hypothese war, dass (1) die operative Versorgung das klinische Ergebnis verbessern würde, (2) ohne Unterschied zwischen den Gruppen.

Material und Methode. In 5 Level-ITraumazentren wurden prospektiv Patienten in die Multizenterstudie einbezogen. Die Stabilisierung erfolgte mittels anatomischer Positionierung einer autologen HamstringSehne als Ersatzplastik der koraokoklavi- kulären und akromioklavikulären Bänder. Die Einheilung des Transplantats wurde durch ein arthroskopisch assistiertes Flaschenzug-Implantat oder mittels temporärer Hakenplatte gesichert. Einschlusskriterien waren hochgradige AC-Gelenkinstabilitäten Typ Rockwood III-V und fehlgeschlagene operative oder konservative Therapie mit einem Mindestintervall von 12 Wochen nach Trauma. Das funktionelle Ergebnis (Subjective Shoulder Value, Constant Score, Taft Score, Acromioclavicular-Joint-Instability Score) wurde präoperativ und bei einer Nachuntersuchung evaluiert. Eine radiologische Auswertung erfolgte prä- und postoperativ sowie bei der Nachuntersuchung.

Ergebnisse. Die Studie erfolgte an 43 Patienten mit einem Alter von 44 \pm 12 (Spanne: 18-64) Jahren; 17 (40\%) Rockwood-Typ III, 26 (60\%) Rockwood-Typ V, 32 (74\%) arthroskopisch assistiert, 11 (26\%) mit Hakenplatte, 35 für Follow-up verfügbar, Follow-up: $12 \pm 3$ (Spanne: 6-20) Monate. Alle klinische Parameter besserten sich signifikant $(p<0,001)$, ohne signifikanten Unterschied zwischen den Gruppen ( $p=$ n. s.) Der CC-Abstand besserte sich von prä- zu postoperativ signifikant (von $19 \pm 5 \mathrm{~mm}$ auf $8 \pm 2 \mathrm{~mm} ; p<0,001$ ), aber es kam zum Repositionsverlust im Follow-up ( $13 \pm 4 \mathrm{~mm}$; $p<0,001)$. Bei 2 Patienten erfolgte nach arthroskopischer Stabilisierung eine Revision. Ein Zweiteingriff zur Materialentfernung wurde bei allen Patienten mit Hakenplatte durchgeführt.

Schlussfolgerung. Die operative Stabilisierung symptomatischer chronischer ACGelenkinstabilitäten verbessert signifikant das klinische Ergebnis im Kurzzeit-Followup. Die funktionellen Ergebnisse zwischen arthroskopisch assistierter Technik und Hakenplatte sind vergleichbar. Dennoch wurde ein gewisser Repositionsverlust beobachtet. Evidenzlevel: IV

Schlüsselwörter Gelenkluxation - Trauma - Transplantate . Schulter - Ligamente 

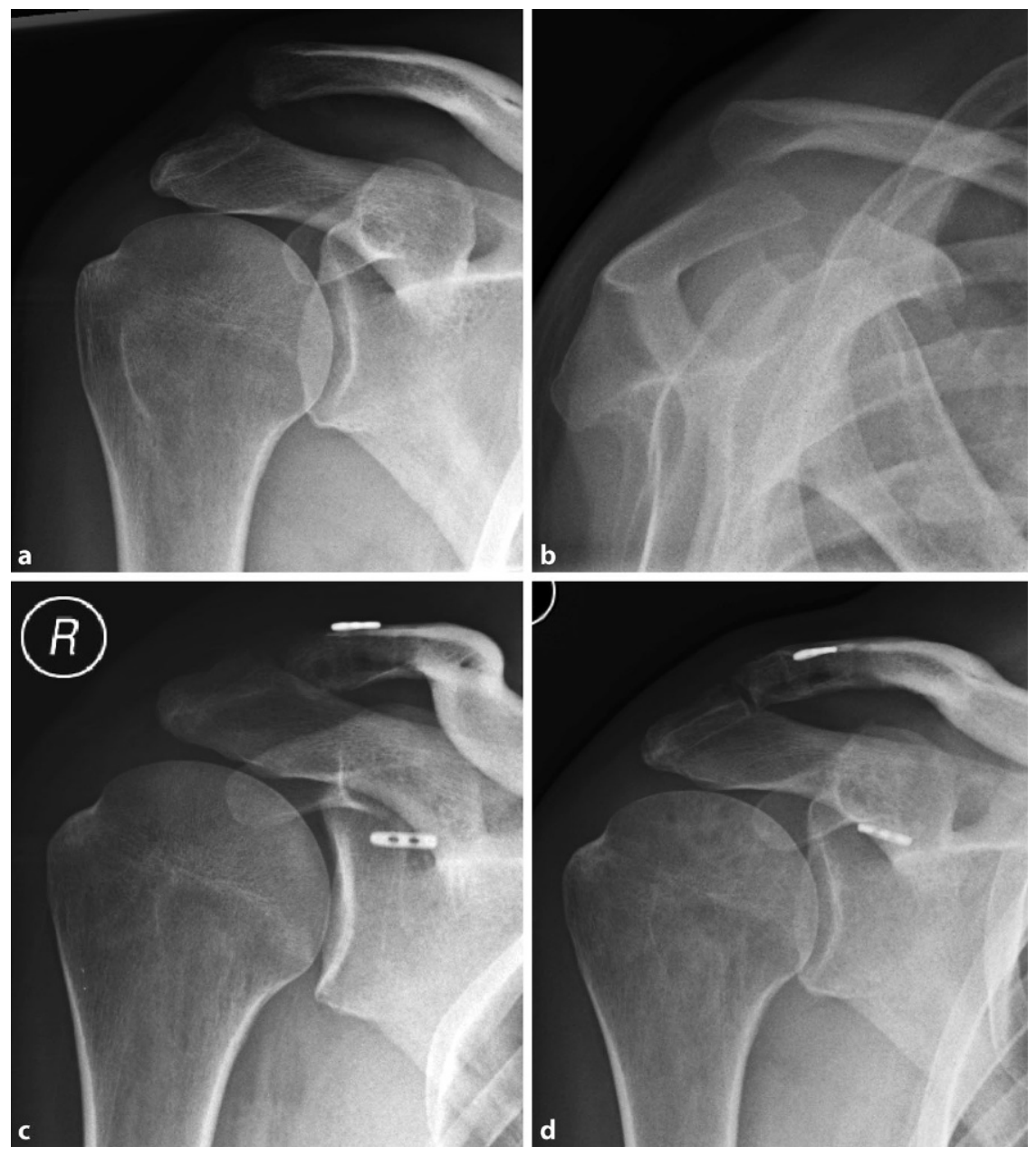

Fig. $2 \Delta$ a Rockwood type III lesion in the anteroposterior view with b horizontal instability in the Alexander view. c Arthroscopically assisted stabilization using a flip-button device with additional tendon graft augmentation. $d$ Follow-up at 9 months

atively, and at a follow-up. Furthermore, a comparison was made with the contralateral shoulder. Horizontal stability was assessed by a further Alexander view at follow-up.

Furthermore, preoperatively performed surgeries or conservative treatments were recorded. Intraoperatively detected concomitant injuries were documented.

\section{Clinical outcome measures}

Functional outcome measures (Subjective Shoulder Value [SSV], Constant Score [CS], Taft Score [TFS], and Acromioclavicular Joint Instability Score [ACJI]) were recorded preoperatively and at follow-up. The TFS and ACJI are AC joint-specific scores evaluating subjective, objective, and radiological value $<0.05$ was considered statistically significant. Statistical analyses were performed using SPSS for Windows (IBM SPSS Statistics 25, Armonk, NY, USA).

The local ethics committee of each clinic approved the study protocol. All work complied with the principles laid down in the Declaration of Helsinki. All included patients provided written informed consent.

\section{Results}

A total of 43 patients were included into this study between February 2011 and November 2013. Mean age was $44 \pm 12$ (range 18-64) years and four patients (9\%) were female. In 21 cases the dominant shoulder was affected. The injury was classified as Rockwood type III in 17 patients (40\%) and Rockwood type $\mathrm{V}$ in 26 patients $(60 \%)$. Of the Rockwood type III patients, 16 had horizontal instability (Rockwood type IIIB). The indication for surgery was due to failed non-operative treatment in 30 patients (70\%), whereas 13 patients (30\%) had had previous surgeries, in some cases multiple. Six patients had previously been stabilized with a hook plate, four with a polydiaxanon suture (PDS) augmentation, two with a double tightrope, and one with a single tightrope. Further revision surgeries were necessary because of an infection. Index surgeries were an arthroscopically assisted technique (group 1) in 32 patients $(74 \%)$ and hook plate stabilization (group 2) in 11 patients (26\%). In group 1, four patients (13\%) had a concomitant intraarticular lesion $(1 \times$ SLAPII lesion, $1 \times$ partial SSC rupture type Fox \& Romeo I, $2 \times$ PASTA lesions). No patient was noted to have an intraarticular lesion in group 2.

Subsequently, 35 patients (81\%) were available for a follow-up (• Table 1). Nine patients refused further participation or were not available for follow-up examinations. Mean follow-up was $12 \pm 3$ (range 6-20) months. All hook plates were removed before the final follow-up examination. All functional outcome measures significantly improved through operative treatment (•Table 2). No difference in the functional result was seen between the two treatment groups ( $\bullet$ Table 3 ). 


\begin{tabular}{l|l|l|l}
\hline \multicolumn{1}{l}{ Table 1} & Comparison of demographic data between the groups at follow-up & \\
\hline & AA group & HP group & p-value \\
\hline Number & 26 & 9 & - \\
\hline Age (years) & $45 \pm 11(18-60)$ & $46 \pm 14(23-60)$ & 0.719 \\
\hline Gender (male:female) & $22: 4$ & $9: 0$ & - \\
\hline Rockwood III & 11 & 7 & 0.121 \\
\hline Rockwood V & 15 & 2 & \\
\hline $\begin{array}{l}\text { Patients with previous surgery (\%) } \\
\text { Follow-up (months) }\end{array}$ & $8(31 \%)$ & $4(44 \%)$ & - \\
\hline$A A$ arthroscopically assisted, HP hook plate & $11 \pm 4(6-18)$ & $13 \pm 4(9-20)$ & 0.208 \\
\hline
\end{tabular}

\begin{tabular}{|c|c|c|c|}
\hline & Preoperative & Follow-up & $p$-value \\
\hline SSV & $49 \pm 18(50-80)$ & $84 \pm 10(60-100)$ & $<0.0001$ \\
\hline CS & $61 \pm 15(63-91)$ & $80 \pm 14(52-98)$ & $<0.0001$ \\
\hline FTS & $6 \pm 1(6-9)$ & $10 \pm 1(7-12)$ & $<0.0001$ \\
\hline ACJ & $31 \pm 13(32-64)$ & $76 \pm 16(29-100)$ & $<0.0001$ \\
\hline
\end{tabular}

Table 3 Comparison of functional outcome measures between arthroscopically assisted treatment and hook plate stabilization at follow-up

\begin{tabular}{llll} 
& Arthroscopically assisted & Hook plate & p-value \\
\hline SSV & $86 \pm 8(70-95)$ & $79 \pm 14(60-100)$ & 0.19 \\
CS & $80 \pm 13(55-98)$ & $81 \pm 17(52-97)$ & 0.96 \\
TFS & $10 \pm 1(7-12)$ & $10 \pm 1(8-12)$ & 0.84 \\
ACJ & $74 \pm 18(29-100)$ & $79 \pm 13(59-100)$ & 0.52 \\
\hline $\begin{array}{l}\text { SSV Subjective Shoulder Value, CS Constant Score, } \\
\text { Instability Score }\end{array}$ &
\end{tabular}

Comparing the clinical results of patients after initially failed operative and nonoperative treatment, there was no statistical significance for SSV $(p=0.294)$, CS $(p=0.682)$, TFS $(p=0.487)$, and ACJI $(p=0.645)$.

Vertical stability was significantly improved by the treatment, reducing the $\mathrm{CC}$ distance from $19 \pm 5 \mathrm{~mm}$ (range 10-28) to $8 \pm 2 \mathrm{~mm}$ (range $4-12 ; p<0.001$ ). There was no difference in CC distance compared to the contralateral shoulder $(10 \pm 2 \mathrm{~mm}$, range $6-13 ; p=0.11)$. The CC distance significantly increased from postoperative to the latest followup $(13 \pm 4 \mathrm{~mm}$, range $6-22 ; p=0.001)$, which was also a significant difference to the contralateral shoulder $(p=0.005)$. Comparing the groups ( $\bullet$ Table 4 ), both improved significantly by treatment (group 1: $p<0.001$; group 2: $p=0.005$ ). However, both groups lost reduction in the follow-up period. For group I the
The overall revision rate was $6 \%$. No infections or fractures, neither of the clavicle nor the coracoid, were observed. Heterotopic ossifications were observed in two patients with arthroscopically assisted stabilization (8\%) and two after a hook plate procedure (22\%).

\section{Discussion}

The aim of this prospective multicenter study was to evaluate clinical and radiological results after treatment of chronic AC joint instability with a hamstring autograft for AC and CC ligament reconstruction. Since data on treatment for stabilization are limited, arthroscopically assisted and hook plate stabilization were compared. Both treatment groups were expected to show comparable clinical and radiological results. Our results showed that operative treatment significantly improved clinical results at short-term follow-up. Comparing the two treatment groups, there was no difference for any of the functional outcome measures. Radiological analysis showed a significantly improved reduction of the AC joint separation after treatment. Nonetheless, reduction was lost over time in both groups, which did not influence the clinical outcome.

The indication for operative intervention in the case of symptomatic chronic AC joint instability is an individual decision based on symptoms, impairments, and the patient's expectations [4]. To date, there is no consensus regarding operative or non-operative treatment for chronic AC joint instability [20, 21]. Regarding operative treatment, multiple techniques have been described for stabilization, all with the idea of AC joint reduction and additional substitution of the ruptured, degenerative CC ligaments. Over the past couple of years, anatomic reconstructions using free tendon grafts have become more and more common for stabilization (about $60 \%$ in the past 3 years) [22]. The torn and degenerative ligaments are biologically augmented by a free tendon graft from the hamstring or tibialis anterior tendon. Free tendon grafts have the advantage of being longer and therefore allow anatomic positioning of the graft. Biomechanically, recon- 


\begin{tabular}{lllll} 
& Preoperative & Postoperative & Follow-up & Contralateral shoulder \\
\hline Arthroscopically assisted & $18 \pm 5(10-27)$ & $8 \pm 2(4-12)$ & $13 \pm 4(6-21)$ & $9 \pm 2(6-13)$ \\
Hook plate & $21 \pm 5(15-30)$ & $10 \pm 4(5-13)$ & $12 \pm 6(6-22)$ & $10 \pm 2(6-13)$
\end{tabular}

struction of the CC ligament complex using a semitendinosus tendon showed a slight decrease in structural properties and stiffness, but was almost comparable to the native situation [23]. Mazzocca et al. also biomechanically tested the highest stability for an anatomic reconstruction using a free tendon graft for the trapezoid and conoid ligaments, which almost fully restored the intact state [24].

Barth et al. evaluated prognostic factors for the treatment of chronic AC joint dislocations. Beside a time to surgery of more than 3 months, persisting horizontal instability due to missing AC stabilization was evaluated as a negative prognostic factor [25]. Scheibel et al. previously analyzed persisting horizontal instability as a negative predictor after AC joint dislocation [5]. Biomechanically, cerclage of the AC joint augmenting the AC capsule significantly improved stability [26, 27]. Therefore, additional AC cerclage should be recommended for treatment of chronic high-grade AC joint instability, as performed in all of our cases. Due to the length of free tendon grafts, additional AC cerclage after CC stabilization is possible using the same graft.

Besides augmenting the CC and AC ligaments, vertical stability has to be restored. For this, either open or arthroscopically assisted techniques are available. Hook plate stabilization is a widely established treatment with rigid and highly stable fixation. Up until now, there have been no clinical results for chronic AC joint instability treated with hook plates. Only McKee has presented his technique for hook plate stabilization in chronic AC joint instability [13]. Regarding treatment of acute AC joint dislocation, Kienast et al. presented the data of 313 patients treated with hook plates. After a mean follow-up of 36 months, TFS averaged at 10.6 points and CS at 92 points [28]. Metzlaff et al. presented the results of 44 patients with a CS of 93 points, TFS of 10.5 points, ACJI of 81 points, and a high return-to- sport rate after 6 months [11]. A slight loss of reduction (less than $50 \%$ of clavicle width) was observed in 2 of 20 (10\%) patients. Further studies confirm a partial loss of reduction in $3-67 \%$ of patients [29-31]. Disadvantages of this treatment are the necessity of implant removal 3-12 months after implantation and the subacromial positioning of the hook, which can result in subacromial injuries and erosions or fractures of the acromion [10, 11, 28].

Tauber et al. published their technique and clinical results of 12 patients with a mean follow-up of 49.5 months [2]. The AC joint was stabilized using a semitendinosus tendon. The graft was pulled through holes in the clavicle and around the coracoid process in a figureof-eight fashion. The ends were wrapped again around the clavicle and the coracoid process and sutured together in the subclavicular space. The graft was protected by a CC cerclage wire or Bosworth screw. The CS improved from 61 to 76 points and pain decreased from 6.2 to 2.3 points. However, implant removal was also necessary with this technique.

Alternatively, the AC joint can be stabilized in arthroscopically assisted techniques using suture-button devices [14, 32]. Implant removal is not required and arthroscopy enables detection and treatment of concomitant intraarticular lesions. Therefore, comparable results are achieved after failed non-operative and operative treatment [21]. However, depending on the technique, a higher risk for horizontal instability can persist.

In 2016, Tauber et al. compared an arthroscopic single- vs. triple-bundle reconstruction [4]. The CS improved significantly for both groups. However, ACJI (85 vs. 58 points) and TFS (10.9 vs. 9.0) were significantly better for the triple-bundle reconstruction. Since clinical outcome is dependent on horizontal stability, this difference regarding AC joint-specific scores resulted from a higher horizontal stability in the triplebundle reconstruction group.

Jensen et al. used a GraftRope ${ }^{\mathrm{TM}}$ system (Arthrex, Naples, FL, USA) with additional horizontal augmentation of the AC joint [12]. The CS averaged at 84 points and the TFS at 9 points. At follow-up, a significantly increased CC distance of plus $3 \mathrm{~mm}$ compared to the contralateral shoulder was observed.

At short-term follow-up, our data confirm the results in the literature, with a significant improvement through operative treatment and good clinical outcomes. Nonetheless, a loss of reduction was observed for both treatment groups, which is comparable to data in the literature [5, 11, 12, 29-31]. Although this result was only significant for the arthroscopically assisted technique, significant differences are also to be expected with an increased number of patients treated with a hook plate. Conversely, the clinical outcome was not influenced. A considerable limitation of hook plate stabilization is the requirement of revision surgery for implant removal after 3 to 12 months. Moreover, an arthroscopically assisted technique enables detection of intraarticular lesions, which can easily go undetected in an open procedure [15]. Independent of the stabilization technique, to improve the clinical outcome, horizontal instability has to be reduced by adding an additional horizontal AC joint augmentation.

Milewski et al. describe high complication rates for CC ligament reconstruction techniques with free tendon grafts for coracoid (in up to 20\%) and clavicular fractures (11\%) [33]. In a current meta-analysis Gowd et al. report infection rates in free tendon transfers for AC joint reconstruction of $7.6 \%$. Neither of these complications were observed in our cohort [8].

Although this is a prospective study comparing two treatment groups, there are some limitations. Follow-up times are quite inhomogeneous, without a minimum follow-up of 2 years. Therefore, 
the data can only be assessed as shortterm results. Furthermore, the comparison between the two treatment groups in terms of functional and radiological outcomes is limited due to the small number of patients treated with hook plates $(n=8)$. Regarding radiological evaluation, measurement accuracy is limited in plain radiographs. Furthermore, cutoff values to define loss of reduction are missing. Therefore, the presented data are just absolute values of the differences in the CC distance. It remains unclear to which extent an increase in CC distance will have a negative effect on clinical outcome.

\section{Conclusion}

Surgical treatment in symptomatic chronic AC joint instability significantly improves clinical outcome in shortterm follow-up. Functional results are comparable between an arthroscopically assisted technique and hook plate stabilization. Nonetheless, some loss of reduction is observed.

\section{Corresponding address}

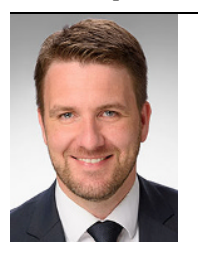

\section{Dr. Gunnar Jensen}

Department for Orthopaedic Surgery and Traumatology, DIAKOVERE Friederikenstift Humboldtstraße 5, 30169 Hannover, Germany Gunnar.jensen@diakovere.de

Acknowledgements. We would like to thank M. Scheibel, N. Kraus-Spiekermann, M. Kriechle, S. Pauly, and J. Dahmen for participation and further patient inclusions into this study.

\section{Compliance with ethical guidelines}

Conflict of interest. A. Ellwein: speaker's honorarium: Arthrex $\mathrm{GmbH}$; H. Lill: consultant: Arthrex GmbH; speaker's honorarium: DePuySynthes; K.-H. Frosch: consultant, research support, and speaker's honorarium: Arthrex $\mathrm{GmbH}$; J. C. Katthagen: research support and speaker's honorarium: Arthrex GmbH; speaker's honorarium: DePuySynthes. M. Jaeger, C. Voigt, C. Schoepp, and G. Jensen declare that they have no competing interests.

The local ethics committee of each clinic approved the study protocol. All procedures performed in studies involving human participants or on human tissue were in accordance with the ethical standards of the institutional and/or national research committee and with the 1975 Helsinki declaration and its later amendments or comparable ethical standards. Informed consent was obtained from all individual participants included in the study.

Open Access. This article is licensed under a Creative Commons Attribution 4.0 International License, which permits use, sharing, adaptation, distribution and reproduction in any medium or format, as long as you give appropriate credit to the original author(s) and the source, provide a link to the Creative Commons licence, and indicate if changes were made. The images or other third party material in this article are included in the article's Creative Commons licence, unless indicated otherwise in a credit line to the material. If material is not included in the article's Creative Commons licence and your intended use is not permitted by statutory regulation or exceeds the permitted use, you will need to obtain permission directly from the copyright holder. To view a copy of this licence, visit http://creativecommons.org/licenses/by/4.0/.

\section{References}

1. Mazzocca AD, Arciero RA, Bicos J (2007) Evaluation and treatment of acromioclavicular joint injuries. Am J Sport Med 35:316-329. https://doi.org/10. 1177/0363546506298022

2. Tauber M, Eppel M, Resch H (2007) Acromioclavicular reconstruction using autogenous semitendinosus tendon graft: results of revision surgery in chronic cases. J Shoulder Elbow Surg 16:429-433. https://doi.org/10.1016/j.jse.2006. 10.009

3. Gumina S, Carbone S, Postacchini F (2009) Scapular dyskinesis and SICK scapula syndrome in patients with chronic type III acromioclavicular dislocation. Arthroscopy 25:40-45. https://doi.org/10.1016/j. arthro.2008.08.019

4. TauberM,VallerD, LichtenbergSetal (2016) Arthroscopic stabilization of chronic acromioclavicular joint dislocations: triple- versus single-bundle reconstruction. Am J Sport Med 44:482-489. https://doi.org/10.1177/0363546515615583

5. Scheibel M, Dröschel S, Gerhardt C, Kraus N (2011) Arthroscopically assisted stabilization of acute high-grade acromioclavicular joint separations. Am J Sport Med 39:1507-1516. https://doi.org/10. 1177/0363546511399379

6. Stein T, Müller D, Blank M et al (2018) Stabilization of acute high-grade acromioclavicular joint separation: a prospective assessment of the clavicular hook plate versus the double double-button suture procedure. Am J Sports Med 46:2725-2734. https://doi.org/10.1177/0363546518788355

7. Andreani L, BonicoliE, ParchiPetal (2014) Acromioclavicular repair using two different techniques. Eur J Orthop Surg Traumatol 24:237-242. https:// doi.org/10.1007/s00590-013-1186-1

8. Gowd AK, Liu JN, Cabarcas BC et al (2018) Current concepts in the operative management of acromioclavicular dislocations: a systematic review and meta-analysis of operative techniques. Am J Sports Med. https://doi.org/10.1177/ 0363546518795147

9. Helfen $T$, Siebenbürger $G$, Ockert $B$, Haasters $F$ (2015) Therapy of acute acromioclavicular joint instability. Meta-analysis of arthroscopic/ minimally invasive versus open procedures.
Unfallchirurg 118:415-426. https://doi.org/10. 1007/s00113-015-0005-z

10. Jensen G, Katthagen JC, Alvarado LE et al (2014) Has the arthroscopically assisted reduction of acute AC joint separations with the double tightrope technique advantages over the clavicular hook plate fixation? Knee Surg Sports Traumatol Arthrosc 22:422-430. https://doi.org/10.1007/ s00167-012-2270-5

11. Metzlaff S, Rosslenbroich S, Forkel PH et al (2016) Surgical treatment of acute acromioclavicular joint dislocations: hook plate versus minimally invasive reconstruction. Knee Surg Sports Traumatol Arthrosc 24:1972-1978. https://doi.org/10.1007/ s00167-014-3294-9

12. Jensen G, Katthagen JC, Alvarado L et al (2013) Arthroscopically assisted stabilization of chronic AC-joint instabilities in GraftRope ${ }^{\mathrm{TM}}$ technique with an additive horizontal tendon augmentation. Arch Orthop Trauma Surg 133:841-851. https:// doi.org/10.1007/s00402-013-1745-2

13. McKee MD (2016) Operative fixation of chronic acromioclavicular joint dislocation with hook plate and modified ligament transfer. J Orthop Trauma 30:S7-S8. https://doi.org/10.1097/BOT. 0000000000000580

14. Braun S, Martetschläger F, Imhoff AB (2014) Arthroscopically assisted reconstruction of acute and chronic AC joint separations. Oper Orthop Traumatol 26:228-236. https://doi.org/10.1007/ s00064-013-0276-X

15. Jensen G, Millett PJ, Tahal DS et al (2017) Concomitant glenohumeral pathologies associated with acute and chronic grade III and grade V acromioclavicular joint injuries. Int Orthop 41:1633-1640. https://doi.org/10.1007/s00264-017-3469-3

16. Kraus N, Gerhardt C, Greiner S, Scheibel M (2010) Arthroskopische Behandlungsmöglichkeiten chronischer Schultereckgelenkinstabilitäten. Arthroskopie 23:293-303. https://doi.org/10. 1007/s00142-010-0578-z

17. Beitzel K, Mazzocca AD, Bak K et al (2014) ISAKOS UpperExtremity Committee Consensus Statement on the Need for Diversification of the Rockwood Classification for Acromioclavicular Joint Injuries. Arthroscopy 30:271-278. https://doi.org/10. 1016/j.arthro.2013.11.005

18. Rockwood CJ (1984) Injuries in the acromioclavicular joint-subluxations and dislocations about the shoulder. In: Rockwood CJ, Green D (eds) Fract. adults. Lippincott, Philadelphia, pp 890-910

19. Alexander O (1949) Dislocation of the acromioclavicular joint. Radiography 15:260

20. Carbone S, Postacchini R, Gumina S (2014) Scapular dyskinesis and SICK syndrome in patients with a chronic type III acromioclavicular dislocation. Results of rehabilitation. Knee Surg Sports Traumatol Arthrosc. https://doi.org/10.1007/ s00167-014-2844-5

21. Kraus N, Hann C, Minkus M et al (2019) Primary versus revision arthroscopically-assisted acromioand coracoclavicular stabilization of chronic ACjoint instability. Arch Orthop Trauma Surg 139:1101-1109. https://doi.org/10.1007/s00402019-03153-3

22. Dyrna F, Berthold DP, Feucht MJ et al (2019) The importance of biomechanical properties in revision acromioclavicular joint stabilization: a scoping review. Knee Surg Sports Traumatol Arthrosc 27(12):3844-3855. https://doi.org/10. 1007/s00167-019-05742-6

23. Costic RS, Labriola JE, Rodosky MW, Debski RE (2004) Biomechanical rationale for development of anatomical reconstructions of coracoclavicular 


\section{Originalarbeit}

ligaments after complete acromioclavicular joint dislocations. Am J Sports Med 32:1929-1936. https://doi.org/10.1177/0363546504264637

24. Mazzocca AD, SantangeloS, Johnson ST et al (2006) A biomechanical evaluation of an anatomical coracoclavicular ligament reconstruction. Am J Sports Med 34:236-246. https://doi.org/10.1177/ 0363546505281795

25. Barth J, Duparc F, Baverel L et al (2015) Prognostic factors to succeed in surgical treatment of chronic acromioclavicular dislocations. Orthop Traumatol Surg Res 101:S305-S311. https://doi.org/10.1016/ j.otsr.2015.09.002

26. Beitzel K, Obopilwe E, Apostolakos J et al (2014) Rotational and translational stability of different methods for direct acromioclavicular ligamentrepair in anatomic acromioclavicularjoint reconstruction. Am J Sports Med 42:2141-2148. https://doi.org/10.1177/0363546514538947

27. Dyrna F, Imhoff FB, Haller B et al (2018) Primary stability of an acromioclavicular joint repair is affected by the type of additional reconstruction of the acromioclavicular capsule. Am J Sports Med 46:3471-3479. https://doi.org/10.1177/ 0363546518807908

28. Kienast B, Thietje R, Queitsch C et al (2011) Midterm results after operative treatment of rockwood grade III-V Acromioclavicular joint dislocations with an AC-hook-plate. Eur J Med Res 16:52-56

29. DeBaets T, Truijen J, Driesen R, Pittevils T (2004) The treatment of acromioclavicular joint dislocation Tossy grade III with a clavicle hook plate. Acta Orthop Belg 70:515-519

30. Koukakis A, Manouras A, Apostolou CD et al (2008) Results using the $\mathrm{AO}$ hook plate for dislocations of the acromioclavicular joint. Expert Rev Med Devices 5:567-572. https://doi.org/10.1586/ 17434440.5.5.567

31. Salem KH, Schmelz A (2009) Treatment of Tossy III acromioclavicular joint injuries using hook plates and ligament suture. JOrthop Trauma 23:565-569. https://doi.org/10.1097/BOT.0b013e3181971b38

32. Braun $S$, Imhoff $A B$, Martetschläger $F$ (2015) Arthroscopically assisted techniques for treatment of acute and chronic acromioclavicular joint injuries Abstract. Unfallchirurg 118:407-414. https://doi.org/10.1007/s00113-015-0004-0

33. Milewski MD, Tompkins M, Giugale JM et al (2012) Complications related to anatomic reconstruction of the coracoclavicular ligaments. Am J Sports Med 40:1628-1634. https://doi.org/10.1177/ 0363546512445273 